\title{
Synthesis and Evaluation of Paclitaxel-Loaded Gold Nanoparticles for Tumor-Targeted Drug Delivery
}

Giulio F. Paciotti, Jielu Zhao, Shugeng Cao, Peggy J. Brodie, Lawrence Tamarkin, Marja Huhta,

Lonnie D. Myer, Jay Friedman, and David G. I. Kingston

\section{Supporting Information}

Cover page 1

${ }^{1} \mathrm{H}$ NMR spectrum of $\mathbf{3}$

${ }^{1} \mathrm{H}$ and ${ }^{13} \mathrm{C}$ NMR spectra of 4

${ }^{1} \mathrm{H}$ and ${ }^{13} \mathrm{C}$ NMR spectra of 5

${ }^{1} \mathrm{H}$ NMR spectrum of 6

$\begin{array}{ll}\text { Figures S1 and S2 } & 6\end{array}$

$\begin{array}{ll}\text { Figure S3 } & 7\end{array}$ 

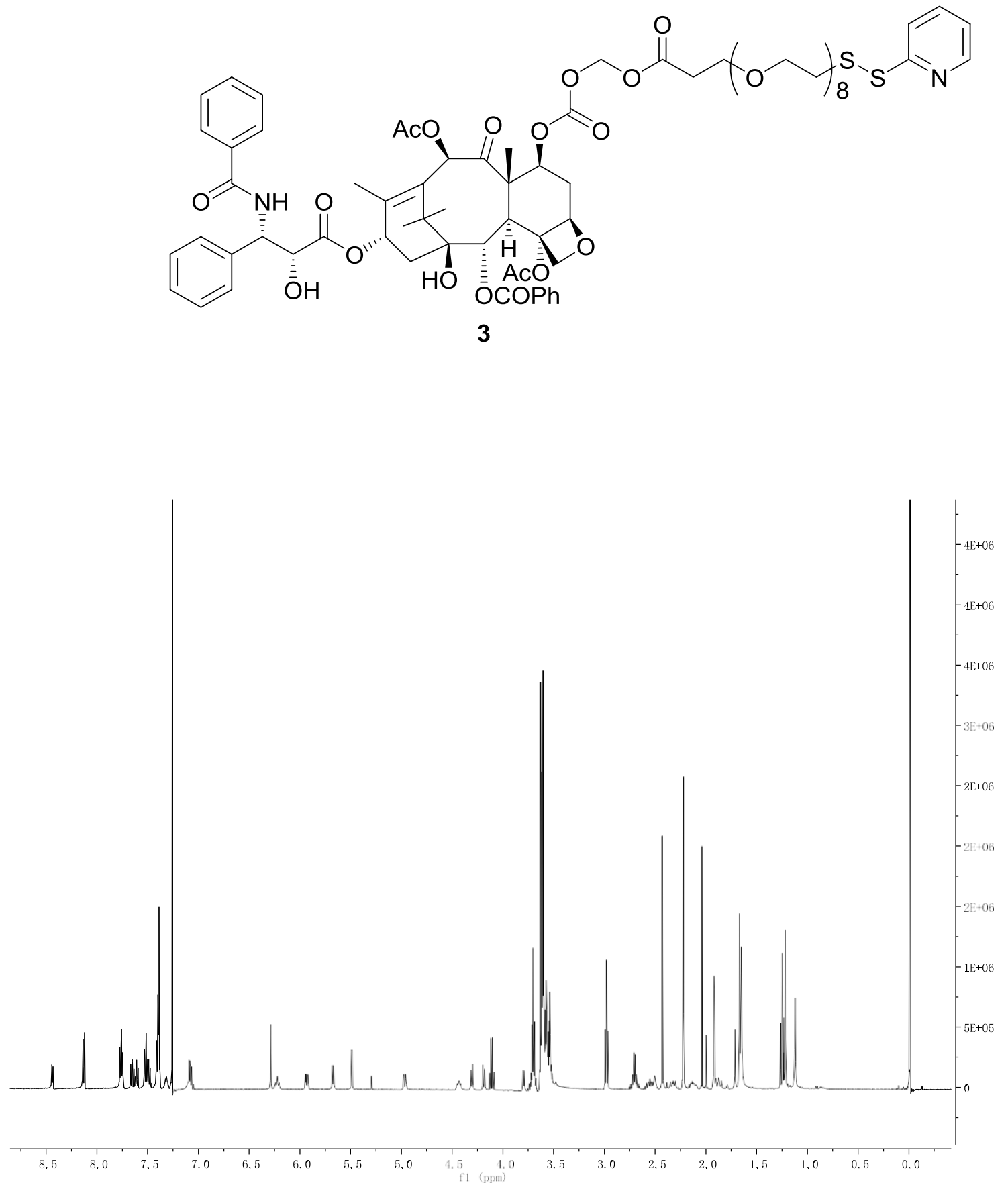


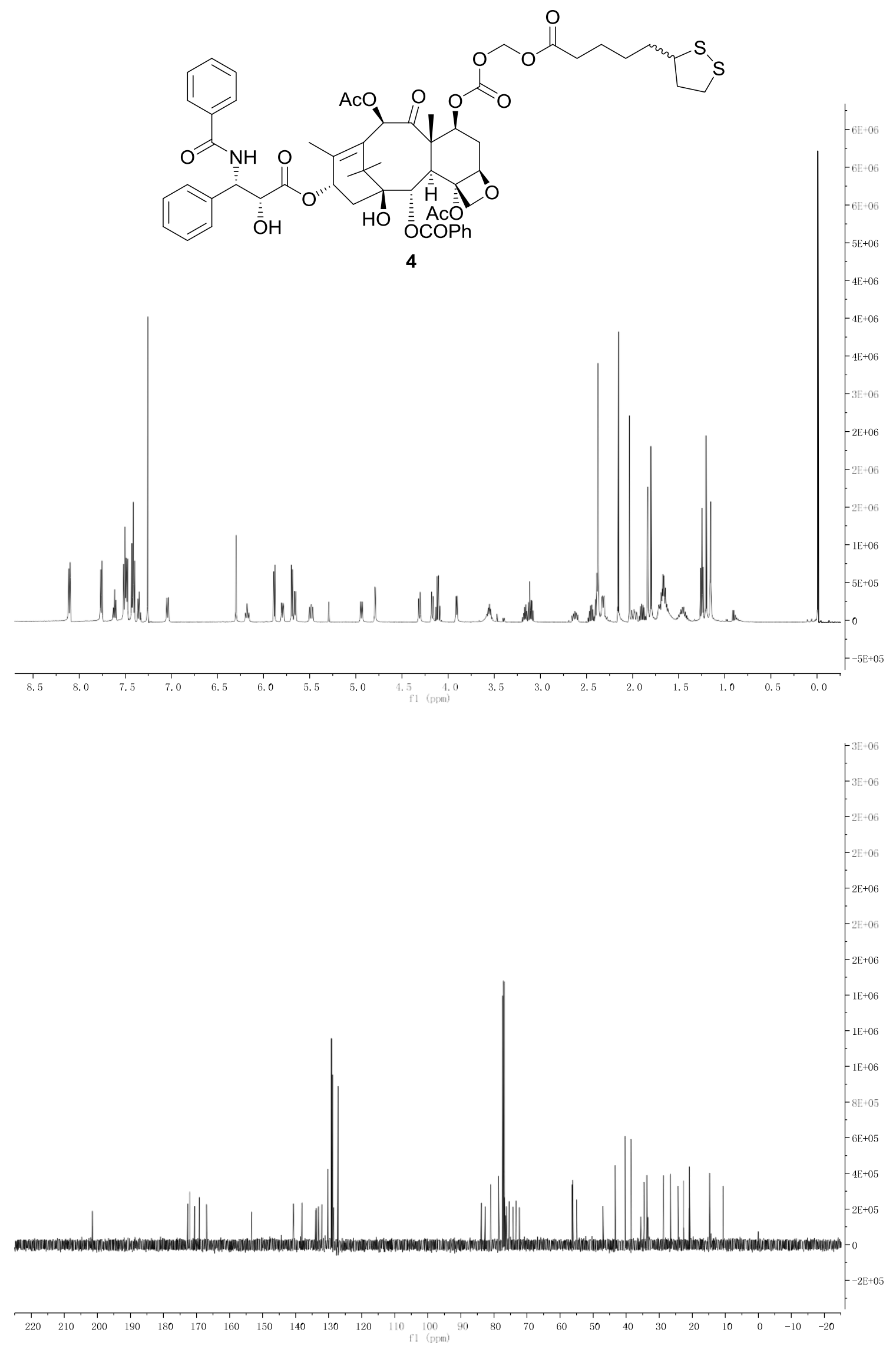



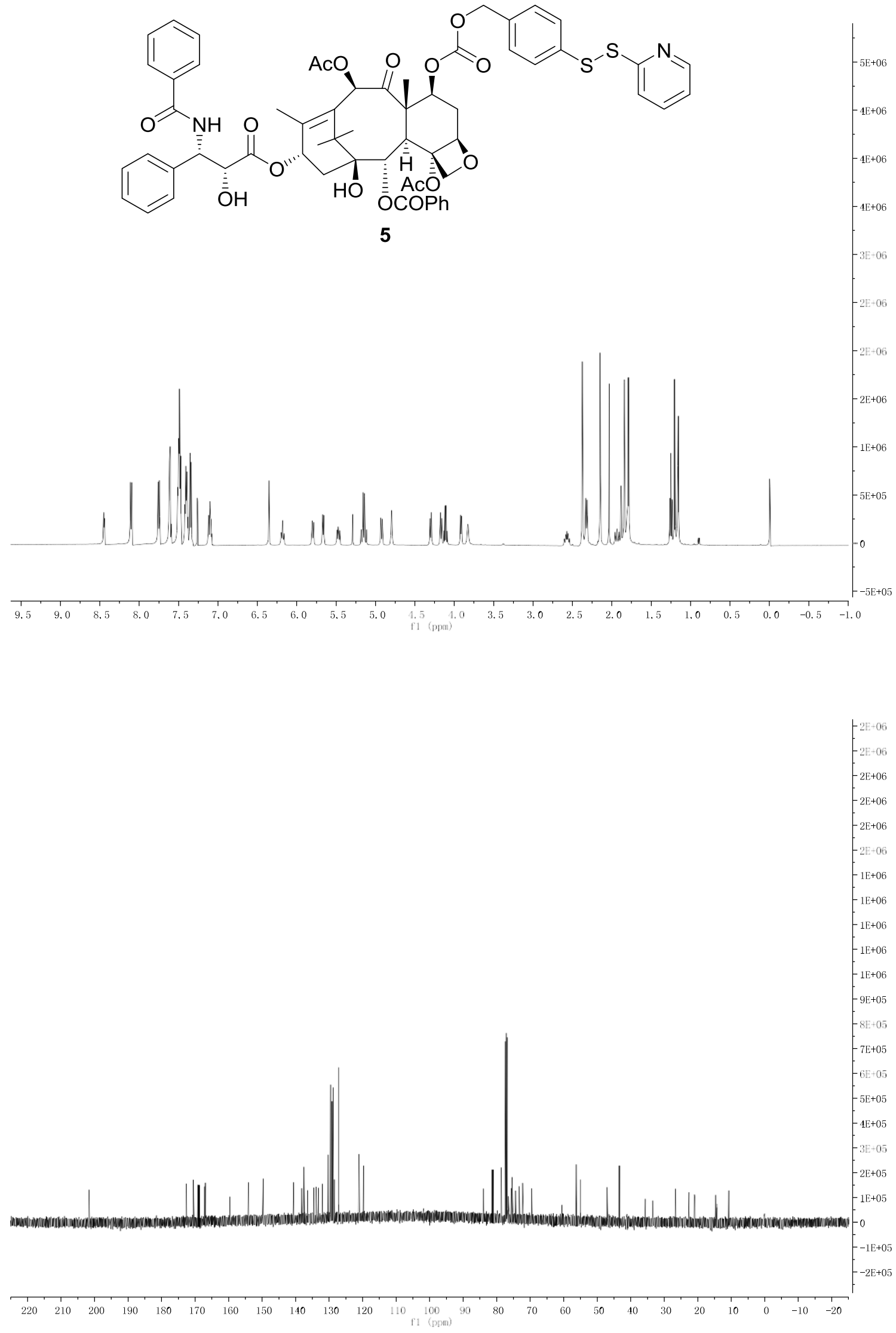

4 

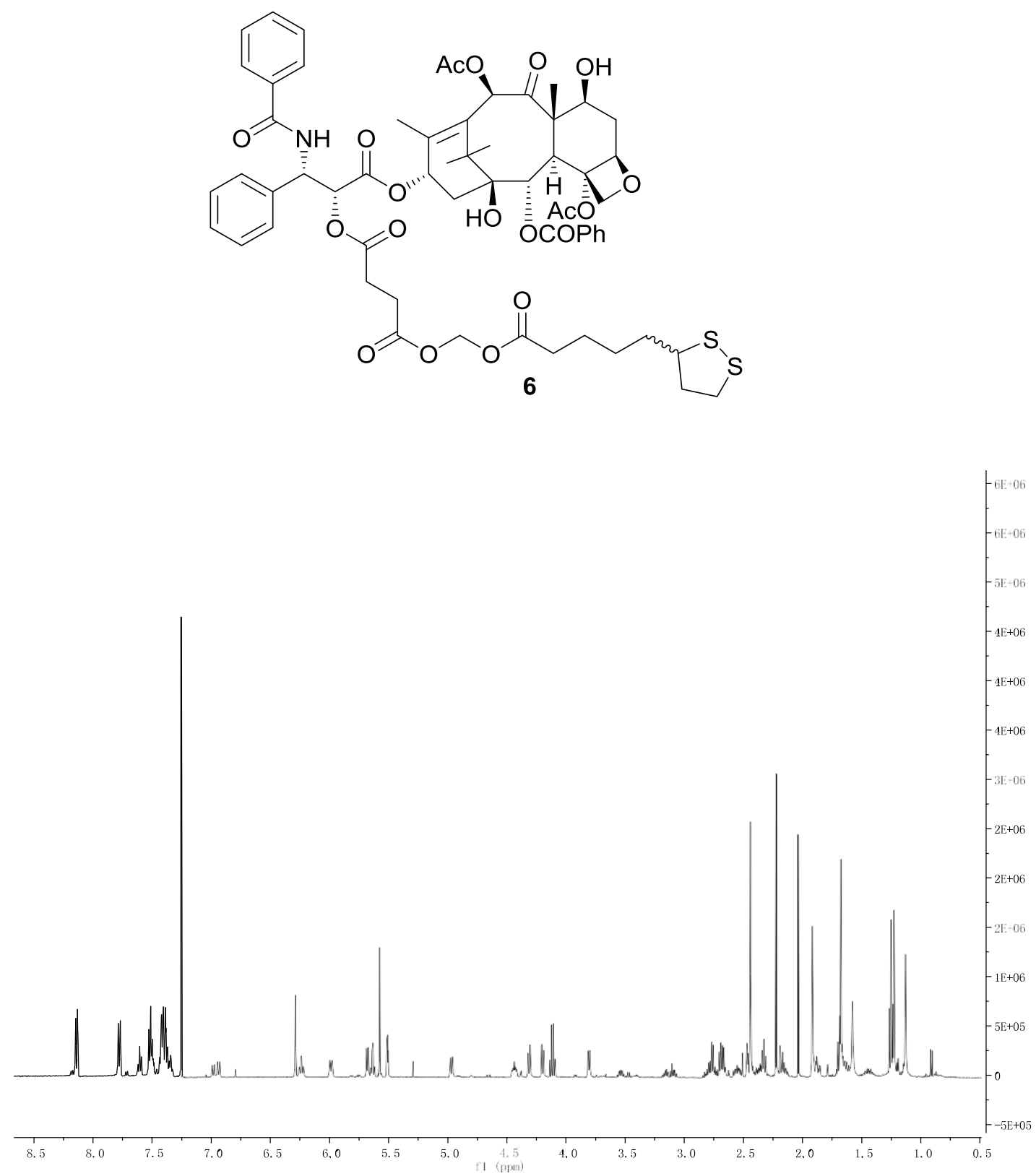


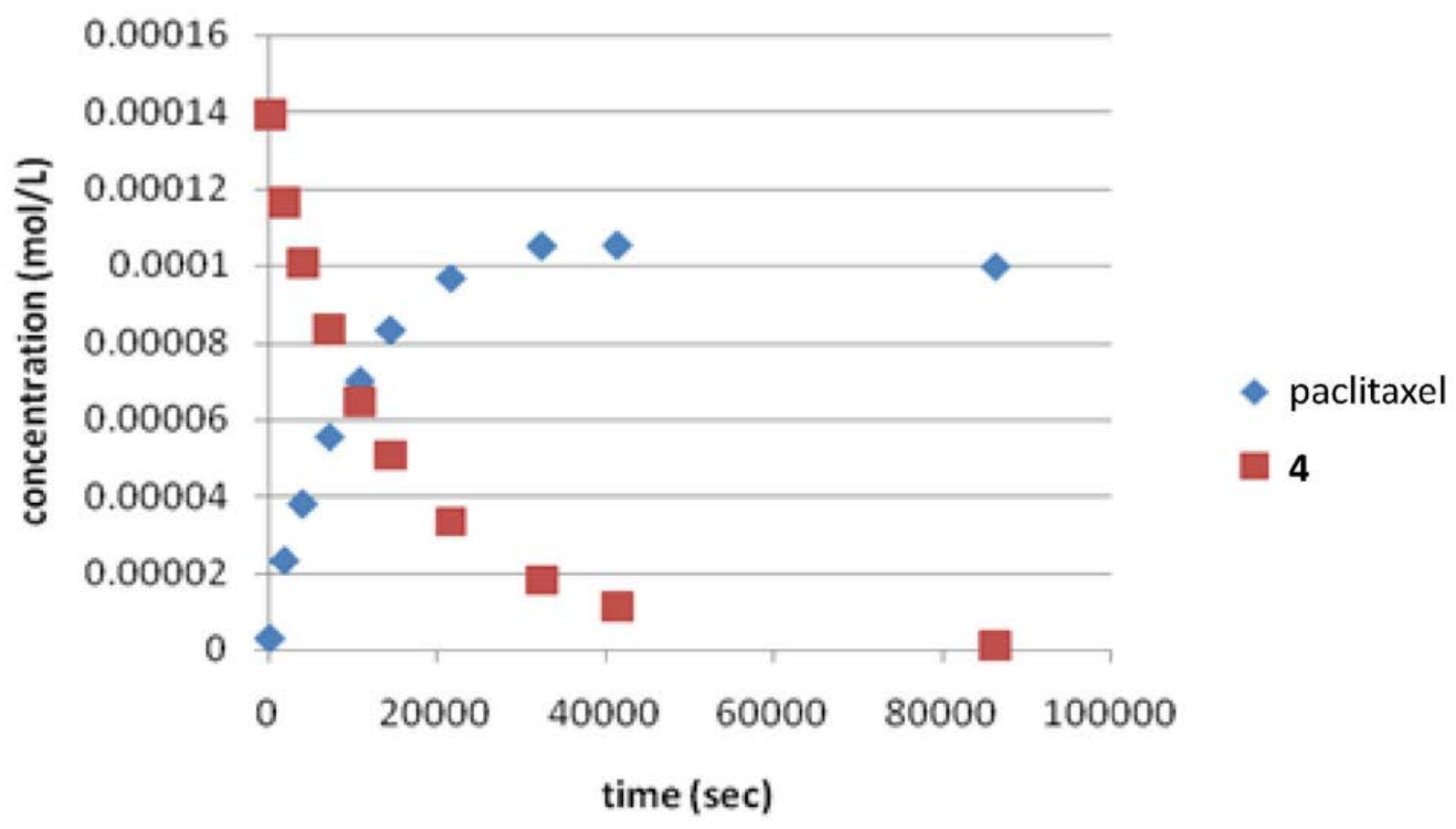

Figure S1. Hydrolytic conversion of 4 to paclitaxel at $\mathrm{pH} 7.4$

A

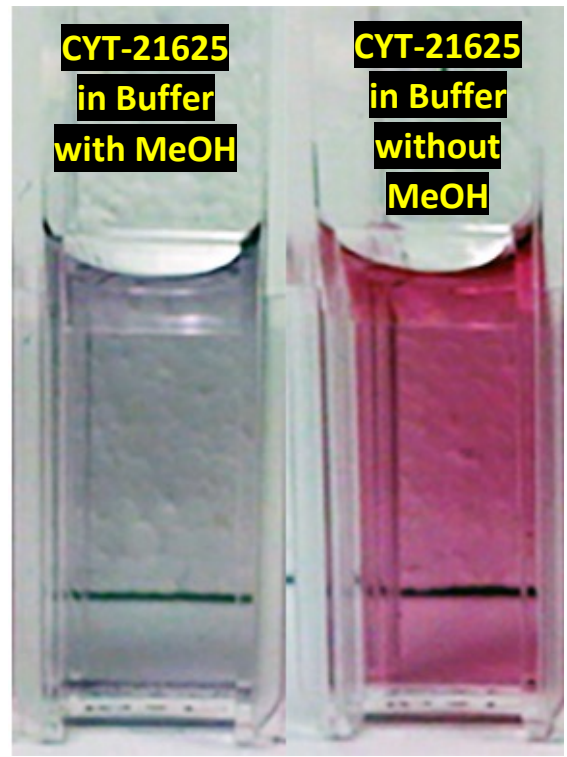

Figure S2. A. Induction of gold nanoparticle agglomeration and precipitation following incubation of CYT-21625 in releasing buffer containing $\mathrm{BME}+50 \% \mathrm{MeOH}$. The image shows that the gold particles (which are typically cherry red in color) undergo agglomeration (the grey/black color; as the particles release surface bound agents (i.e., compound 5). B. The same experiment conducted in the absence of $\mathrm{MeOH}$. Here the particles appear to be monodispersed and are not undergoing agglomeration. 


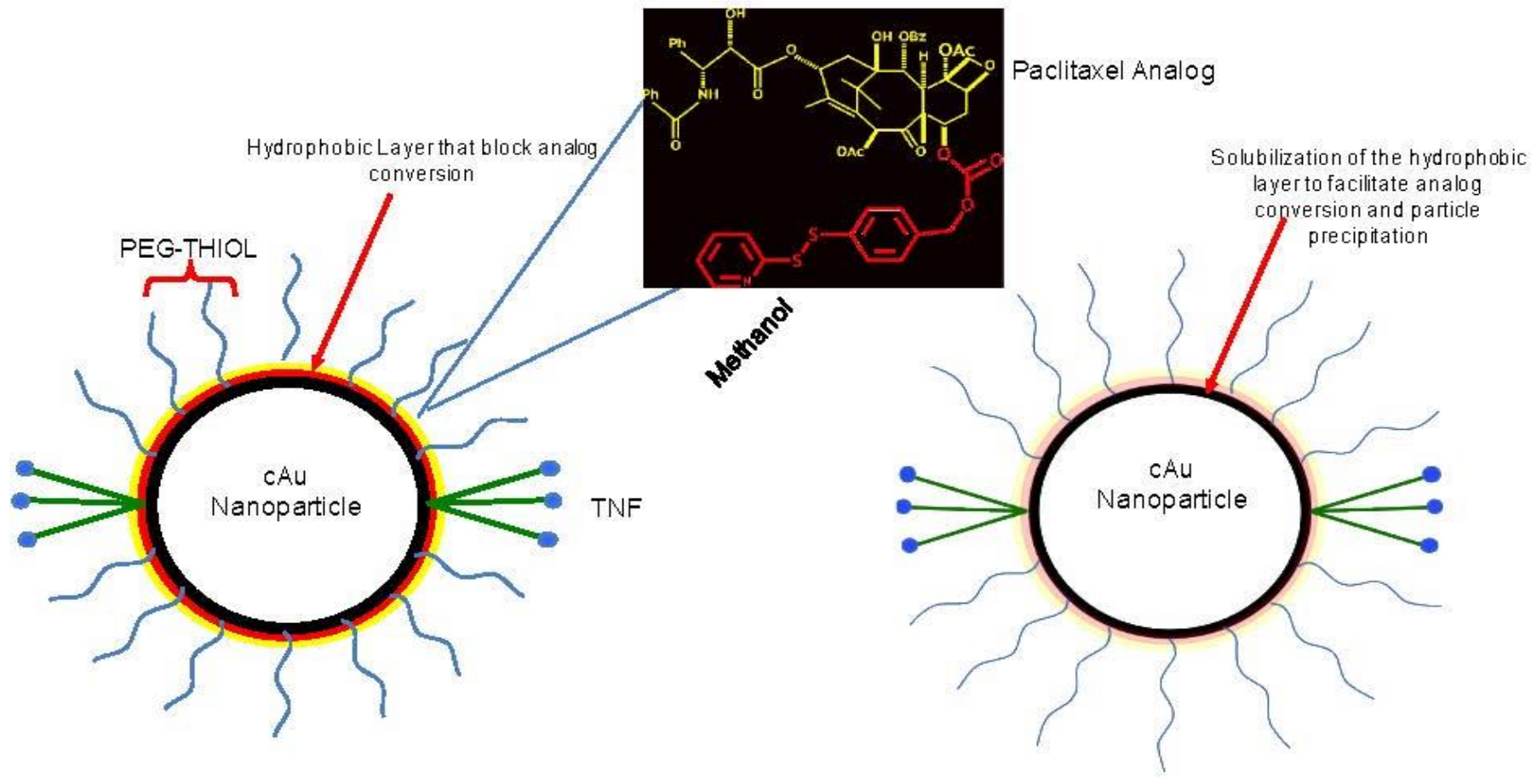

Figure S3. Hypothetical hydrophobic layer of paclitaxel on the gold nanoparticle surface reducing susceptibility to hydrolytic or reductive conversion to paclitaxel. Addition of $\mathrm{MeOH}$ solubilizes this layer and facilitates paclitaxel release. 